\title{
BMJ Open Pulmonary rehabilitation for pneumoconiosis: protocol for a systematic review and meta-analysis
}

\author{
Hulei Zhao, ${ }^{1,2,3}$ Yang Xie, ${ }^{2,3}$ Jiajia Wang, ${ }^{3}$ Xuanlin $\mathrm{Li}^{3}$ Jiansheng $\mathrm{Li}^{3}$
}

To cite: Zhao H, Xie Y, Wang J, et al. Pulmonary rehabilitation for pneumoconiosis: protocol for a systematic review and meta-analysis. BMJ Open 2019;9:e025891. doi:10.1136/ bmjopen-2018-025891

- Prepublication history for this paper is available online. To view these files please visit the journal online (http://dx.doi. org/10.1136/bmjopen-2018025891).

Received 07 August 2018 Revised 04 July 2019 Accepted 30 July 2019

Check for updates

(c) Author(s) (or their employer(s)) 2019. Re-use permitted under CC BY-NC. No commercial re-use. See rights and permissions. Published by BMJ.

${ }^{1}$ Longhua Hospital Shanghai University of Traditional Chinese Medicine, Shanghai University of Traditional Chinese Medicine, Shanghai, China

${ }^{2}$ Department of Respiratory Diseases, The First Affiliated Hospital of Henan University of Chinese Medicine, Zhengzhou, China

${ }^{3}$ Collaborative Innovation Center for Respiratory Disease Diagnosis and Treatment \& Chinese Medicine Development of Henan Province, Henan University of Chinese Medicine Zhengzhou, China

Correspondence to

Dr Yang Xie;

xieyanghn@163.com

\section{ABSTRACT}

Introduction Pneumoconiosis is characterised by diffuse fibrosis in lung tissue, and its incidence is on the rise. At present, there are limited therapeutic options for pneumoconiosis. Pulmonary rehabilitation (PR) has been widely used to treat pneumoconiosis, however, there is limited evidence concerning its efficacy. Therefore, we plan to conduct a systematic review to investigate the efficacy and safety of PR for pneumoconiosis.

Methods and analysis The following databases will be searched from their inception to 1 April 2019: PubMed, Embase, The Cochrane Library, Web of Science, Chinese Biomedical Literature Database, China National Knowledge Infrastructure, Chongqing VIP and Wanfang Data. Randomised controlled trials of PR for pneumoconiosis will be included. Primary outcomes will include 6 min walk distance and St. George's Respiratory Questionnaire. Study selection, extraction of data and assessment of study quality each will be independently undertaken. Statistical analysis will be conducted using Review Manager software.

Ethics and dissemination This systematic review will provide up-to-date information on PR for pneumoconiosis. The review does not require ethical approval and will be disseminated electronically through a peer-reviewed publication or conference presentations. PROSPERO registration number CRD42018095266.

\section{INTRODUCTION}

Pneumoconiosis is a disease characterised by diffuse fibrosis in lung tissue, and is mainly caused by long-term inhalation of productive mineral dust in occupational activities. ${ }^{1-3}$ Dust exposure in coal mining has been one of the main occupational hazard factors in China over time, and coal worker pneumoconiosis (CWP) and silicosis are the main types of the 12 kinds of national occupational pneumoconiosis. $^{34}$ In 2016, the National Occupational Disease Report shows that China has reported a total of 31789 cases of occupational diseases, including 27992 cases of occupational pneumoconiosis accounted for $88.06 \%$ of the total cases reported. ${ }^{5}$ The incidence of pneumoconiosis is on the rise. ${ }^{6-8}$ In China, the cumulative incidence of pneumoconiosis in state-owned coal mines ranges from $4 \%$ to

\section{Strengths and limitations of this study}

This systematic review aims to evaluate the efficacy and safety of pulmonary rehabilitation for pneumoconiosis.

- The review methods were carefully planned to minimise risk of selective bias, reporting bias and publication bias in the completed review according to current guidelines and prospectively registered with the International Prospective Register of Systematic Reviews.

- The search strategy for this review is broad and comprehensive, including studies from multiple electronic databases.

- Limitations may include issues of poor reporting affecting risk of bias assessment and confidence in results.

$17 \% .^{9}$ A systematic analysis of studies from 2001 to 2011 showed that the pooled prevalence of CWP was $6.02 \%$ in China, which was higher compared with UK $(0.8 \%$, 1998$2000)$ and the USA $(3.2 \%$ in $2000 \mathrm{~s}) .{ }^{10}$ During 1999-2016, a total of 38358 years of potential life lost to life expectancy (mean per decedent $=8.8$ years) and 2707 years of potential life lost before age 65 years (mean per decedent $=7.3$ years) were attributed to CWP. ${ }^{11}$ Long-term silica dust exposure is associated with substantially increased mortality among Chinese workers. $^{12}$

At present, there are limited therapeutic options for pneumoconiosis. ${ }^{13}$ Pulmonary rehabilitation $(\mathrm{PR})$ is a comprehensive intervention based on a thorough patient assessment followed by patient-tailored therapies that include, but are not limited to, exercise training, education, social support and behavioural change, which are designed to improve the physical and psychological condition of people with chronic respiratory disease and promote the long-term adherence to health-enhancing behaviours. ${ }^{14}$ Evidencebased support for PR in the management of patients with chronic respiratory disease has grown tremendously, and this comprehensive 
intervention has been clearly demonstrated to reduce dyspnoea, increase exercise performance and improve health-related quality of life (HRQL). ${ }^{15}$ As an effective intervention, PR could provide sustained improvement of functional capacity and reduce healthcare utilisation for occupational respiratory diseases. ${ }^{16}$ Recently, PR has been widely used to treat pneumoconiosis. ${ }^{16-18}$ However, there is limited evidence concerning its efficacy for pneumoconiosis patients. Therefore, we plan to conduct a systematic review to investigate the efficacy and safety of PR for pneumoconiosis.

\section{METHODS AND ANALYSIS \\ Study type}

All published randomised controlled trials (RCTs) with a parallel, cluster or cross-over design will be included.

\section{Participants}

Pneumoconiosis patients diagnosed by relevant standard (such as GBZ 70-2015 National occupational health standards $)^{3}$ will be included. There will be no restrictions on age, sex, ethnicity, education or economic status. We will exclude studies including participants with complications, such as pulmonary heart disease, tuberculosis and chronic respiratory failure.

\section{Interventions}

The intervention we focus on in this review is a comprehensive PR, which is based on exercise training, including or not including health education, nutritional intervention and psychosocial support.

Comparisons investigated are:

- PR versus no treatments;

- PR adjunctive to other treatments versus other treatments alone.

\section{Outcome measures}

\section{- Primary outcomes}

The primary outcome measures were functional capacity and HRQL, as measured by $6 \mathrm{~min}$ walk distance, ${ }^{16}{ }^{19-21}$ St. George's Respiratory Questionnaire. ${ }^{162122}$

- Secondary outcomes

The secondary outcomes measures were pulmonary function, symptoms, acute exacerbations and adverse events, as measured by forced vital capacity, forced expiratory volume in the $1 \mathrm{~s},{ }^{21} 22$ the modified Medical Research Council dyspnoea scale,${ }^{23}$ frequency of acute exacerbations ${ }^{1624}$ and incidence of adverse events.

\section{Search methods}

We will search PubMed, Embase, The Cochrane Library, Web of Science, Chinese Biomedical Literature Database, China National Knowledge Infrastructure, Chongqing VIP and Wanfang Data from their inception to 1 April 2019. We have developed detailed search strategies for each electronic database without language restrictions to attempt to identify all eligible studies. We will also review

\begin{tabular}{|c|c|}
\hline No & No search terms \\
\hline$\# 1$ & Pneumoconiosis [MeSH Terms] \\
\hline \#2 & pneumoconiosis [Title/Abstract] \\
\hline$\# 3$ & Asbestosis [MeSH Terms] \\
\hline$\# 4$ & asbestosis [Title/Abstract] \\
\hline$\# 5$ & Silicosiss [MeSH Terms] \\
\hline \#6 & silicosis [Title/Abstract] \\
\hline$\# 7$ & Anthracosis [MeSH Terms] \\
\hline \#8 & Anthracosis [Title/Abstract] \\
\hline \#9 & Anthracosilicosis [MeSH Terms] \\
\hline$\# 10$ & Anthracosilicosis [Title/Abstract] \\
\hline$\# 11$ & $\begin{array}{l}\text { \#1 OR \#2 OR \#3 OR \#4 OR \#5 OR \#6 OR \#7 OR } \\
\text { \#8 OR \#9 OR \#10 }\end{array}$ \\
\hline$\# 12$ & rehabilitation [MeSH Terms] \\
\hline$\# 13$ & rehabilitation [Title/Abstract] \\
\hline$\# 14$ & health education [MeSH Terms] \\
\hline \#15 & health education [Title/Abstract] \\
\hline$\# 16$ & psychological counselingTitle/Abstract] \\
\hline$\# 17$ & nutritional guidance [Title/Abstract] \\
\hline$\# 18$ & Baduanjin [Title/Abstract] \\
\hline$\# 19$ & eight-section brocade [Title/Abstract] \\
\hline \#20 & respiratory training [Title/Abstract] \\
\hline \#21 & sports training [Title/Abstract] \\
\hline \#22 & exercise therapy [MeSH Terms] \\
\hline$\# 23$ & exercise therapy [Title/Abstract] \\
\hline \#24 & physical fitness [MeSH Terms] \\
\hline \#25 & physical fitness [Title/Abstract] \\
\hline \#26 & physical exertion [MeSH Terms] \\
\hline \#27 & physical exertion [Title/Abstract] \\
\hline \#28 & kinesiotherapy [Title/Abstract] \\
\hline \#29 & muscle training [MeSH Terms] \\
\hline \#30 & muscle training [Title/Abstract] \\
\hline \#31 & physical endurance [MeSH Terms] \\
\hline \#32 & physical endurance [Title/Abstract] \\
\hline \#33 & $\begin{array}{l}\# 12 \text { OR \#13 OR \#14 OR \#15 OR \#16 OR \#17 OR } \\
\text { \#18 OR \#19 OR \#20 OR \#21 OR \#22 OR \#23 OR } \\
\text { \#24 OR \#25 OR \#26 OR \#27 OR \#28 OR \#29 OR } \\
\text { \#30 OR \#31 OR \#32 }\end{array}$ \\
\hline \#34 & \#11 AND \#33 \\
\hline
\end{tabular}

This search strategy will be modified as required for other electronic databases.

the reference lists of included studies or relevant systematic reviews to identify any potentially eligible studies. The search strategy for PubMed is shown in table 1.

\section{Searching other resources}

We will conduct a search on the website of ClinicalTrials. gov, WHO International Clinical Trials Registry platform 


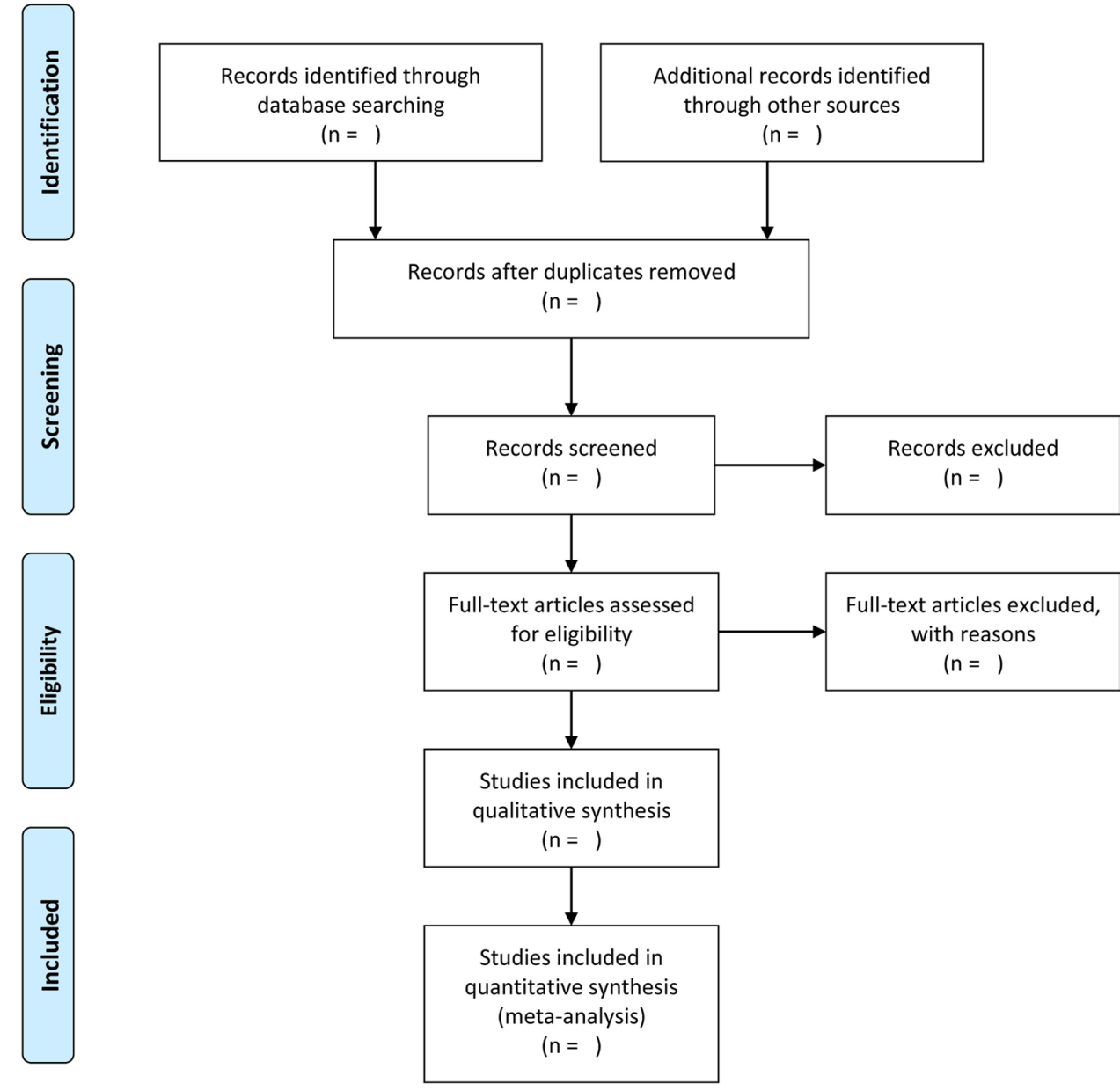

From: Moher D, Liberati A, Tetzlaff J, Altman DG, The PRISMA Group (2009). Preferred Reporting /tems for Systematic Reviews and MetaAnalyses: The PRISMA Statement. PLoS Med 6(6): e1000097. doi:10.1371/journal.pmed1000097

Figure 1 PRISMA flow diagram.

and Chinese Clinical Trial Registry, Conference Proceedings Index (Web of Science Core Collection) to identify additional ongoing or unpublished studies.

\section{Study selection}

Two review authors (JW and XL) will independently examine titles and abstracts retrieved from the search and select all potentially eligible studies. Then these full-text articles will be obtained and the same review authors will review them independently according to the inclusion criteria. We will resolve all disagreements by consensus, and a third review author ( $\mathrm{HZ}$ ) will act as an arbiter when consensus cannot be reached. The review authors will record all full texts that do not meet the inclusion criteria and provide the rationale for their exclusion. We will count multiple publications reporting the same group of participants or their subsets as one single study. Details of the selection process are presented in figure 1 .

\section{Data extraction and management}

Two investigators (JW and XL) will independently extract data from included studies. A structured and standardised data extraction form will be used to extract the relevant information. We will complete a data extraction sheet for every study included in the review, involving information on details of authors, year of publication, study design, characteristics of participants, intervention, comparator and outcomes.

Included studies with greater than $20 \%$ attrition will be considered at high risk of attrition bias. ${ }^{25}$ When SDs of the change of included studies are missing, we will substitute for them the mean SD of other included studies. ${ }^{26}$ We will exclude from the analysis studies, in which only medians and percentiles are available and there are no means of calculating mean change scores. ${ }^{26}$

\section{Quality assessment and analysis}

Methodological quality will be independently assessed using the Cochrane Collaboration's tool for assessing 
risk of bias in RCTs. ${ }^{27}$ The assessment details include: sequence generation, allocation concealment, blinding of participants and personnel, blinding of outcome assessors, incomplete outcome data, selective reporting and other sources of bias. Each domain will be assessed as 'low risk', 'high risk' or 'unclear risk' according to the description details of eligible studies. Two review authors (JW and XL) will complete the data extraction and score each study, with a third review author (HZ) acting as an arbiter when differences occur between JW and XL. We will summarise the risk of bias and settle differences in author interpretation of data through discussion.

\section{Data analysis}

We plan to pool data from the outcomes of each study to provide an overall measure of the effect. For dichotomous outcome data, we will present the effect using relative risks with $95 \%$ CI. For continuous data, we will present the effect using mean differences with $95 \%$ CI. We will convert the unit to the commonly used one, when different units of measurement are employed. For example, we will convert the 'month' or 'year' into 'weeks'.

We plan to use a $\chi^{2}$ test to estimate heterogeneity. Further analysis can be performed using the $\mathrm{I}^{2}$ statistic. A random-effect model will be used to interpret the results if heterogeneity is statistically significant, whereas a fixed-effect model will be used if heterogeneity is not statistically significant. We will regard heterogeneity as substantial when $\mathrm{I}^{2}$ is greater than $50 \%$ or a low $\mathrm{p}$ value $(<0.01)$ is reported for the $\chi^{2}$ test for heterogeneity. ${ }^{28}$

When 10 or more studies are included in the meta-analysis, we will investigate reporting biases (such as publication bias) by using funnel plots. ${ }^{27}$

We will undertake statistical analysis by using Review Manager software. ${ }^{29}$ As with cross-over trials, we will consider only the first phase and exclude from the analysis data obtained during the second phase (ie, up until the point of crossover).

\section{Subgroup analysis}

If data permit, we plan to conduct subgroup analyses for different intervention forms to assess whether the treatment effects are different in different subgroups. Different intervention forms refer to whether exercise training is combined with other rehabilitation measures, such as health education, nutritional intervention and psychosocial support.

\section{Sensitivity analysis}

We will conduct sensitivity analyses to explore the robustness of the findings regarding the study quality and sample size. We will exclude studies one by one and comparing the results in the analysis. ${ }^{30}$ Sensitivity analyses will be showed in a summary table.

\section{Quality of evidence assessment}

The quality assessment of the body of evidence is performed to determine the extent to which an estimate of effect is close to the true quantity/value, that is, it is not distorted by internal or external bias within and across studies. The assessment will be conducted by outcome of interest using the Grading of Recommendations Assessment, Development and Evaluation system. ${ }^{31}$ The quality of outcome measures will be categorised as high, moderate, low and very low.

\section{Patient and public involvement}

Patients were not involved in the development of the research question or the design of this study at this stage.

The procedure for this protocol will be conducted according to the guidance provided by the Preferred Reporting Item for Systematic Reviews and Meta-analyses protocols. ${ }^{32}$

\section{Potential amendments}

We do not envisage any further amendments to this protocol. However, in case of any changes, the amendment shall be detailed out in the final report.

\section{DISCUSSION}

Pneumoconiosis is a disease with no end to treatment, there are limited therapeutic options. As a comprehensive treatment, PR has been proved to improve the quality of life of patients with respiratory diseases (eg, chronic obstructive pulmonary disease, asthma and idiopathic pulmonary interstitial fibrosis). ${ }^{33-35}$ At present, PR has been used in the treatment of pneumoconiosis, and the efficacy of PR for patients with pneumoconiosis was already evaluated by meta-analysis of RCTs. ${ }^{36}$ However, due to the inaccuracy of a small number of trials, the small number of participants, and the indirectness of evidence, the quality of the evidence is low. Therefore, it is necessary to make an update. The objective of this study is to provide a protocol of systematic review and meta-analysis to update PR for pneumoconiosis. This systematic review will provide a detailed summary of the current evidences related to the efficacy and safety of PR in improving breathlessness, exercise limitation and health status impairment of pneumoconiosis patients. This evidence may be useful to clinicians, patients and health policy-makers with regard to the use of PR in pneumoconiosis treatment.

\section{Ethics and dissemination}

This systematic review will provide up-to-date information on PR for pneumoconiosis. This review will be disseminated electronically through a peer-reviewed publication or conference presentations.

Contributors $\mathrm{HZ}$ drafted the protocol and will arbitrate any disagreements. YX and $\mathrm{JL}$ conceived the study and revised the manuscript. JW and XL will independently screen the potential studies, extract data from the included studies and assess the risk of bias. $\mathrm{HZ}$ will search the literature and conduct the data analysis. All authors have read and approved the final manuscript for publication.

Funding This work was supported by the Henan Province Priority and Advantage Discipline Construction Engineering Projects-Traditional Chinese Medicine (No. STS-ZYX-2017025); Program for Science and Technology Innovation Talents in 
Universities of Henan Province (No.19HASTIT008); National Public Welfare Industry Research Project-Traditional Chinese Medicine Industry (No.201507001-01); Special subject of Chinese medicine research of Henan Province (2018ZY1003).

Disclaimer The funder had no role in the development of the protocol.

Competing interests None declared.

Patient consent for publication Not required.

Ethics approval This review does not require ethical approval.

Provenance and peer review Not commissioned; externally peer reviewed.

Open access This is an open access article distributed in accordance with the Creative Commons Attribution Non Commercial (CC BY-NC 4.0) license, which permits others to distribute, remix, adapt, build upon this work non-commercially, and license their derivative works on different terms, provided the original work is properly cited, appropriate credit is given, any changes made indicated, and the use is non-commercial. See: http://creativecommons.org/licenses/by-nc/4.0/.

\section{REFERENCES}

1. Laney AS, Petsonk EL, Hale JM, et al. Potential determinants of coal workers' pneumoconiosis, advanced pneumoconiosis, and progressive massive fibrosis among underground coal miners in the United States, 2005-2009. Am J Public Health 2012;102(Suppl 2):S279-83.

2. Cullinan P, Reid P. Pneumoconiosis. Prim Care Respir J 2013;22:249-52.

3. National Health and Family Planning Commission of the People's Republic of China. GBZ 70-2015 National occupational health standards

4. Han L, Gao Q, Yang J, et al. Survival Analysis of Coal Workers' Pneumoconiosis (CWP) Patients in a State-Owned Mine in the East of China from 1963 to 2014. Int J Environ Res Public Health 2017:14:489.

5. National Health Commission of the People's Republic of China. About 2015-2016 the occupational-disease-prevention work situation; 2018

6. Li Y, Tang XL, Xiao XB. New research progress of pneumoconiosis therapy. Occup Health 2013;29:2561-3.

7. Zhang M, Wang D, Zheng Y-dong, et al. [Analyses on the characteristics and the trends of pneumoconiosis notified between 1997 and 2009, in China]. Zhonghua Lao Dong Wei Sheng Zhi Ye Bing Za Zhi 2013;31:321.

8. Blackley DJ, Halldin CN, Laney AS. Continued increase in prevalence of coal workers' pneumoconiosis in the United States, 1970-2017. Am J Public Health 2018;108:1220-2.

9. Cui K, Shen F, Han B, et al. Comparison of the cumulative incidence rates of coal workers' pneumoconiosis between 1970 and 2013 among four State-Owned colliery groups in China. Int $J$ Environ Res Public Health 2015;12:7444-56.

10. Mo J, Wang L, Au W, et al. Prevalence of coal workers' pneumoconiosis in China: a systematic analysis of 2001-2011 studies. Int J Hyg Environ Health 2014;217:46-51.

11. Mazurek JM, Wood J, Blackley DJ, et al. Coal Workers' Pneumoconiosis-Attributable Years of Potential Life Lost to Life Expectancy and Potential Life Lost Before Age 65 Years - United States, 1999-2016. MMWR Morb Mortal Wkly Rep 2018;67:819-24.

12. Chen $\mathrm{W}$, Liu $\mathrm{Y}$, Wang $\mathrm{H}$, et al. Long-Term exposure to silica dust and risk of total and cause-specific mortality in Chinese workers: a cohort study. PLOS Med 2012;9:e1001206.

13. Liang $\mathrm{YX}$, Wong $\mathrm{O}, \mathrm{Fu} \mathrm{H}$, et al. The economic burden of pneumoconiosis in China. Occup Environ Med 2003;60:383-4.
14. Celli BR, Decramer M, Wedzicha JA, et al. An official American thoracic society/ European respiratory Society statement. Eur Respir $J$ 2016;45:879-905.

15. Nici L, Donner C, Wouters E, et al. American thoracic Society/ European respiratory Society statement on pulmonary rehabilitation. Am J Respir Crit Care Med 2006;173:1390-413.

16. Ochmann U, Kotschy-Lang N, Raab W, et al. Long-Term efficacy of pulmonary rehabilitation in patients with occupational respiratory diseases. Respiration 2012;84:396-405.

17. Tsang EW, Kwok H, Chan AKY, et al. Outcomes of community-based and home-based pulmonary rehabilitation for pneumoconiosis patients: a retrospective study. BMC Pulm Med 2018;18:133.

18. Ochmann U, Kotschy-Lang N, Raab W, et al. Occupational respiratory diseases and pulmonary rehabilitation. Atemwegs Lungenkr 2014:40:164-79.

19. Vaz M, Soares F, Van Zeller M, et al. Six-minute walk distance improvement in patients with pseudo-tumoral silicosis after a pulmonary rehabilitation program. Eur Respir J 2013;42.

20. Noh SR. Availability of the 6-min walk test in coal workers pneumoconiosis evaluations. Chest 2010;137:1492-3.

21. Dowman LM, McDonald CF, Hill CJ, et al. The evidence of benefits of exercise training in interstitial lung disease: a randomised controlled trial. Thorax 2017;72:610-9.

22. Liu L, Li Z. Health education in the treatment and rehabilitation of pneumoconiosis. Zhonghua Lao Dong Wei Sheng Zhi Ye Bing Za Zhi 2015;33:62-4.

23. Dale MT, Mckeough ZJ, Troosters T, et al. Exercise training to improve exercise capacity and quality of life in people with nonmalignant dust-related respiratory diseases: the Cochrane library. John Wiley \& Sons, Ltd, 2011.

24. Hou CC, Cao DF, Gao P, et al. [Preliminary study on the evaluation of pneumoconiosis]. Zhonghua Lao Dong Wei Sheng Zhi Ye Bing Za Zhi 2018;36:749-52.

25. Lacasse $Y$, Brosseau L, Milne $S$, et al. Pulmonary rehabilitation for chronic obstructive pulmonary disease. Cochrane Database Syst Rev 2002;88:720-1.

26. McCarthy B, Casey D, Devane D, et al. Pulmonary rehabilitation for chronic obstructive pulmonary disease. Cochrane Database Syst Rev 2015;(2):CD003793.

27. Higgins JPT, Green S. Cochrane Handbook for systematic reviews of interventions version 5.1.0. Wiley-Blackwell, 2011.

28. Higgins JPT, Thompson SG, Deeks JJ, et al. Measuring inconsistency in meta-analyses. BMJ 2003;327:557-60.

29. Clarke M, Oxman A. Review Manager (RevMan) [Computer program]. Version 5.0. 2008.

30. Cai W, Stewart R, Mueller C, et al. Poststroke depression and risk of stroke recurrence and mortality: protocol of a meta-analysis and systematic review. BMJ Open 2018;8:e026316.

31. Guyatt GH, Oxman AD, Vist GE, et al. GRADE: an emerging consensus on rating quality of evidence and strength of recommendations. BMJ 2008;336:924-6.

32. Moher D, Shamseer L, Clarke M, et al. Preferred reporting items for systematic review and meta-analysis protocols (PRISMA-P) 2015 statement. BMJ 2015;349:97647-g47.

33. Zhang H-L, Li J-S, Yu X-Q, et al. An evaluation of activity tolerance, patient-reported outcomes and satisfaction with the effectiveness of pulmonary daoyin on patients with chronic obstructive pulmonary disease. Int J Chron Obstruct Pulmon Dis 2017;12:2333-42.

34. Yu X, Li X, Wang L, et al. Pulmonary rehabilitation for exercise tolerance and quality of life in IPF patients: a systematic review and meta-analysis. Biomed Res Int 2019;2019:8498603

35. Osadnik CR, Singh S. Pulmonary rehabilitation for obstructive lung disease. Respirology 2019;21.

36. Dale MT, McKeough ZJ, Troosters T, et al. Exercise training to improve exercise capacity and quality of life in people with nonmalignant dust-related respiratory diseases. Cochrane Database Syst Rev 2015;14. 